\title{
Static impurities in a supersolid of interacting hard-core bosons on a triangular lattice
}

\author{
Xue-Feng Zhang, ${ }^{1,2, *}$ Yu-Chuan Wen, ${ }^{3}$ and Sebastian Eggert ${ }^{1}$ \\ ${ }^{1}$ Physics Dept. and Res. Center OPTIMAS, Univ. of Kaiserslautern, 67663 Kaiserslautern, Germany \\ ${ }^{2}$ Institute of Theoretical Physics, Chinese Academy of Sciences, P.O. Box 2735, Beijing 100190, China \\ ${ }^{3}$ Center of Theoretical Physics, Dept. of Physics, Capital Normal Univ., Beijing 10048, China
}

\begin{abstract}
We study the effect of impurities in a supersolid phase in comparison to the behavior in the solid and superfluid phases. A supersolid phase has been established for interacting hardcore bosons on a triangular lattice which may be realizable by ultracold atomic gases. Static vacancies are considered in this model which always lower the magnitude of the order parameter in the solid or superfluid phases. In the supersolid phase, however, the impurities directly affect both order parameters simultaneously and thereby reveal an interesting interplay between them. In particular the solid order may be enhanced at the cost of a strong reduction of the superfluidity, which shows that the two order parameters cannot be in a simple superposition. We also observe an unusual impurity pinning effect in the solid ordered phase, which results in two distinct states separated by a first-order transition.
\end{abstract}

PACS numbers: $67.80 . \mathrm{kb}, 75.10 . \mathrm{Jm}, 05.30 . \mathrm{Jp}$

A bosonic supersolid phase is characterized by the coexistence of two seemingly contradictory order parameters, a solid crystalline order and a superfluid density. This reflects the spontaneous breaking of two independent symmetries, namely translation and a $\mathrm{U}(1)$ gauge rotation, which are also known as diagonal and offdiagonal order, respectively. The simultaneous breaking of two independent symmetries in the supersolid phase is counter-intuitive and unusual, because normally a spontaneously broken order locks the system into a single phase. Only when the remaining fluctuations are large enough, two independent order parameters may exist in one phase, e.g. due to frustration. Having been predicted forty years ago, $\frac{1}{2}$ supersolids recently received renewed interest after a possible observation in ${ }^{4} \mathrm{He}, \stackrel{2}{2}$ The presence of mobile ${ }^{3} \mathrm{He}$ impurities appears to be important in those systems, which are predicted to raise $T_{c}$ but reduce the superfluid density $\underline{\underline{3}}$ While the experiments are still controversially discussed, $\stackrel{4}{=}$ there is now very strong numerical evidence that a supersolid phase is realized for interacting hardcore bosons on a triangular lattice..$^{-11}$ Such a model can potentially be realized by ultracold atoms in optical traps $\stackrel{12}{=}$ Tunable superexchange models have already been experimentally created in this rapidly emerging field $\frac{13}{3}$ and interacting hard core bosons $\frac{14}{14}$ have also been discussed. Most recently also triangular lattices have been possible $\frac{15}{\underline{15}}$ so it appears likely that supersolidity will soon be a central topic for hard core boson models in the ultra-cold gases community.

While the coexistence of the corresponding two order parameters is well established numerically in the hard core boson systems,$\frac{6}{2}$ the microscopic interplay between them is still unclear. We now study the impurity effects on both order parameters simultaneously in the supersolid phase, in order to clarify if the two order parameters are in a simple superposition or how they may interact locally. The use of substitutional impurities in strongly correlated systems has become a standard tool for understanding the underlying quantum phases $\stackrel{16-19}{-1 n}$ par- ticular, it is possible to study local expectations values around defects 20,21 for an analysis of the elementary excitations and direct comparison with theoretical models. In the supersolid phase we are now able to consider the effect of static impurities on two coexisting order parameters simultaneously.

The model we will consider in this paper is the spin- $1 / 2$ model on a triangular lattice

$$
H=-t \sum_{\langle i j\rangle}\left(\hat{S}_{i}^{+} \hat{S}_{j}^{-}+\text {h.c. }\right)+V \sum_{\langle i j\rangle} \hat{S}_{i}^{z} \hat{S}_{j}^{z}-B \sum_{i} \hat{S}_{i}^{z}
$$

with antiferromagnetic exchange $V$ in the z-direction corresponding to nearest neighbor repulsion and ferromagnetic exchange $t$ in the $\mathrm{x}$-y-directions, corresponding to the kinetic energy and $B=\mu-3 V$ in terms of the chemical potential of the equivalent hard-core boson problem. The simplest impurities are given by lattice vacancies in model (1) $:^{22}$

The two order parameters in the supersolid are given by the structure factor $S(q=[4 / 3 \pi, 0])=$ $\left\langle\left|\sum_{r} \hat{S}_{r}^{z} e^{i q \cdot r}\right|^{2}\right\rangle / N^{2}$ for the solid order, and by the superfluid density $\rho_{s}$, which is typically measured using the winding number $W$ in quantum Monte Carlo (QMC)

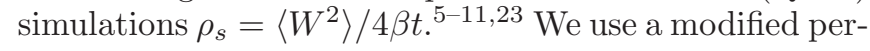
turbation theory and the directed-loop stochastic series expansion QMC algorithm $\underline{24}$ with finite size scaling up to $N=324$ sites at a temperature of $T=0.02 \mathrm{~V}$. In order to avoid trapping in one of the degenerate states it is also essential to implement parallel tempering in the parameter space $\stackrel{25,26}{\underline{2}}$

For reference we first examine a single vacancy in the solid phase, which already shows interesting effects. As shown in the inset of Fig. 1 this phase occurs for small xy-coupling $t$. The solid order is characterized by a $2 / 3$ filled state for positive fields with exactly two spins on each triangle pointing up. For negative fields there is an equivalent $1 / 3$ filled ordered phase due to the spin flip symmetry around $B=0$. The vacancy in the XXZ 


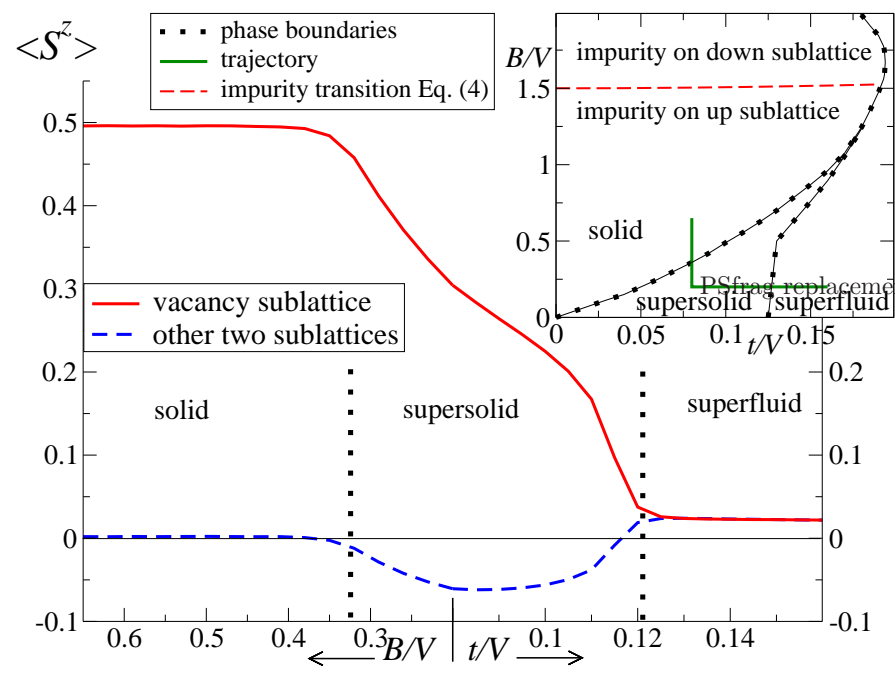

FIG. 1: (color online). The average magnetic moment of the sublattice which contains the vacancy (solid) and the other two sublattices (dashed). Inset: The phase diagram with the impurity phases and the plot trajectory we use in Figs. 13 and 5 along $t / V=0.08$ and then along $B / V=0.2$.

model (11) does not break this symmetry, ${ }^{22}$ so it is sufficient to consider only positive fields $B>0$ in the phase diagram in Fig. 1. The choice of the spin-down sublattice (pointing against the field) gives a three-fold degeneracy, which is however lifted by the vacancy. In particular, for $0<B<1.5 \mathrm{~V}$ and $t=0$ the vacancy site must belong to one of the spin-up sublattices, as can be seen by simple energetic considerations. Therefore the order in the entire system is pinned by a single defect and only a twofold degeneracy remains. The average occupation $\left\langle S^{z}\right\rangle$ on the different sublattices in Fig. 1 shows that this pinning also continues throughout the supersolid phase. However, the spin density of the other two sublattices surprisingly point against the field in the supersolid phase.

For larger fields $B>1.5 \mathrm{~V}$ there is a transition to a different state, where the order is now pinned on the opposite sublattice with no remaining degeneracy. Therefore, a single impurity can in fact induce a transition between two distinct ordered states of the entire system. The transition line also depends on the xy-coupling $t$ as can be seen by perturbation theory in the "hopping" terms $H_{i j}=-t\left(\hat{S}_{i}^{+} \hat{S}_{j}^{-}+\right.$h.c. $)$. Unfortunately, the usual perturbative correction to the wavefunction $|\psi\rangle \approx|0\rangle+\sum_{\langle i j\rangle}|i j\rangle\left\langle i j\left|H_{i j}\right| 0\right\rangle /\left(E_{0}-E_{i j}\right)$ diverges with the number of hopping terms, i.e. the system size $N$. Here $|0\rangle$ is the ordered state and $|i j\rangle$ has opposite spins exchanged on the bond $i, j$ relative to $|0\rangle$. Of course the number of lattice sites $N$ must be irrelevant in the ordered phase, so the trick is to modify the perturbation correction to include only those hopping terms which actually affect a local expectation value 14 For example, to calculate the energy correction at one bond $\delta E_{i j}$ only the

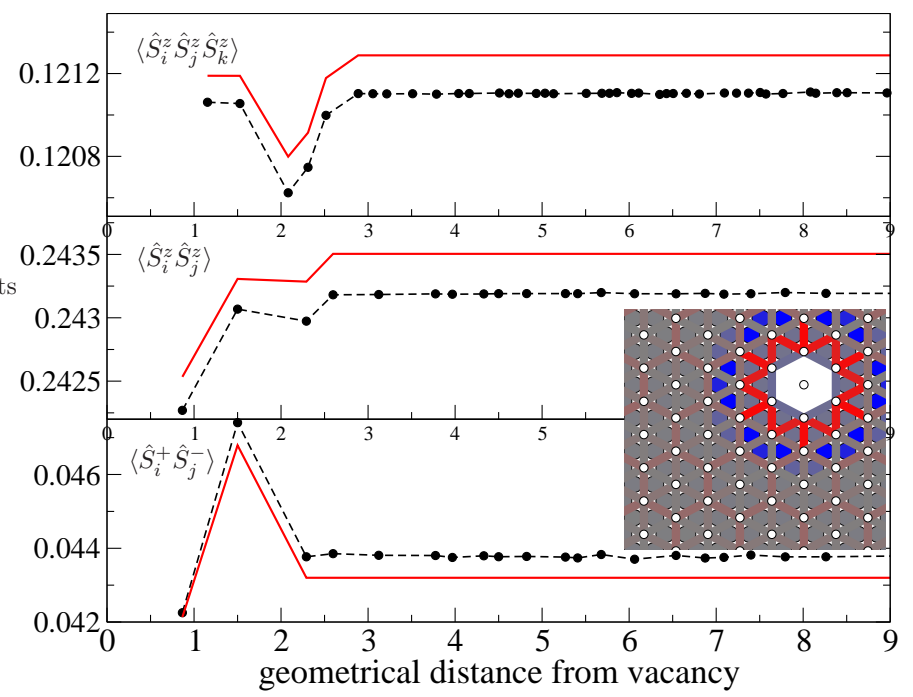

FIG. 2: (color online). (a) $\left\langle\hat{S}_{i}^{z} \hat{S}_{j}^{z} \hat{S}_{k}^{z}\right\rangle$ with $i, j, k$ on one triangle, (b) $\left\langle\hat{S}_{i}^{z} \hat{S}_{j}^{z}\right\rangle$ and (c) $\left\langle\hat{S}_{i}^{+} \hat{S}_{j}^{-}\right\rangle$, where $i, j$ are neighboring sites on sublattices not occupied by the impurity as a function of distance in the solid phase $(t / V=0.08, B / V=0.65$, $N=144$ ). The modified perturbation theory (solid) agrees very well with the QMC results (dashed). Inset: Distribution of $\left\langle\hat{S}_{i}^{+} \hat{S}_{j}^{-}\right\rangle$and $\left\langle\hat{S}_{i}^{z} \hat{S}_{j}^{z} \hat{S}_{k}^{z}\right\rangle$ on the bonds and triangles, respectively. Red signals an increase and blue a decrease relative to the gray bulk values.

corresponding hopping term is considered

$$
\left|\psi_{i j}\right\rangle \approx|0\rangle+|i j\rangle\left\langle i j\left|H_{i j}\right| 0\right\rangle /\left(E_{0}-E_{i j}\right)
$$

and we simply get

$$
\delta E_{i j}=\left|\left\langle i j\left|H_{i j}\right| 0\right\rangle\right|^{2} /\left(E_{0}-E_{i j}\right)=t^{2} /\left(E_{0}-E_{i j}\right) .
$$

For the case of a vacancy, the excitation energy $E_{i j}$ depends on the location of the bond and it also depends on which sublattice is pointing down in the ordered state $|0\rangle$. After summing over all contributions, we find that the energy difference between the two possible pinned ordered states is given by $\Delta E=B-\frac{3 V}{2}-\frac{7 t^{2}}{10 V}+\mathcal{O}\left(t^{3}\right)$. Therefore, the impurity driven transition line runs along

$$
B \approx 3 V / 2+7 t^{2} / 10 V
$$

as shown in the inset of Fig. 1, which also agrees with our numerical QMC results.

Using the modified perturbation theory with a restricted sum in Eq. (2) it is also possible to analytically calculate local expectation values, e.g. when calculating $\left\langle\hat{S}_{i}^{z}\right\rangle$ all hopping terms connecting to the site $i$ are included. The results give a good indication about the local order around the vacancies. In particular, $\left\langle\hat{S}_{i}^{z} \hat{S}_{j}^{z}\right\rangle$ and $\left\langle\hat{S}_{i}^{z} \hat{S}_{j}^{z} \hat{S}_{k}^{z}\right\rangle$ with $i, j, k$ on neighboring sites are good indicators of the local solid order, which are reduced around the vacancy. On the other hand the quantum fluctuations $\left\langle\hat{S}_{i}^{+} \hat{S}_{j}^{-}\right\rangle$on bonds are enhanced as shown in Fig. 2. Interestingly, the order reduction is not correlated in space 


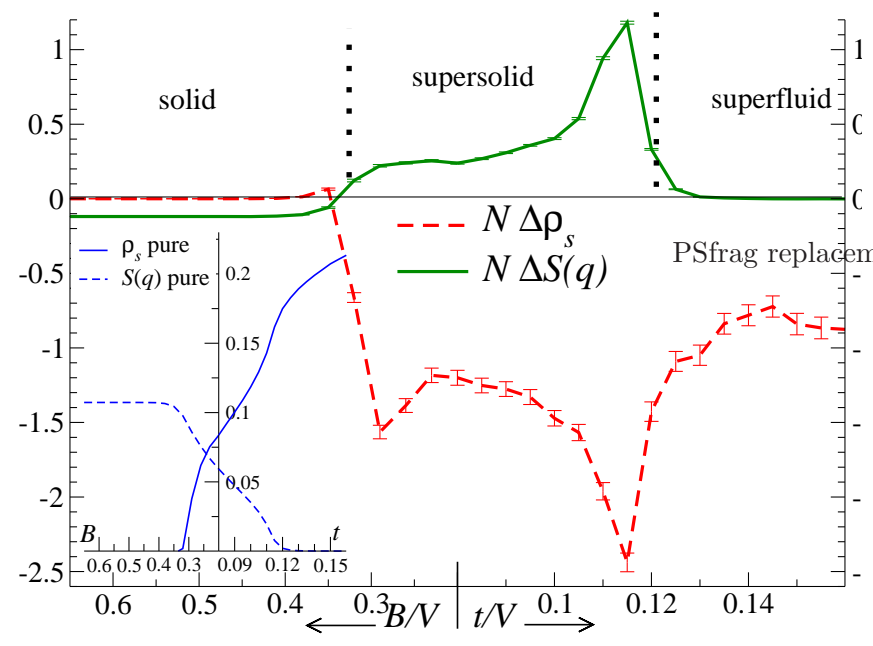

FIG. 3: (color online). Scaled impurity corrections to the superfluid density $N \Delta \rho_{s}$ and the structure factor $N \Delta S(q)$. Inset: Order parameters in a pure system.

with the fluctuation enhancement and the effect is also not always strongest directly at the vacancy. The inset in Fig. 2 shows the distribution of the local expectation values around the impurity on the lattice directly. The expectation values $\left\langle\hat{S}_{i}^{+} \hat{S}_{j}^{-}\right\rangle$between two non-impurity sublattices is different from those bonds involving the impurity sublattice even very far from the impurity. This is a secondary effect from the pinned order and should not be mistaken for an independent bond order.

It is not surprising that an impurity generally reduces the order parameter locally. The main question for the supersolid phase is now if the vacancy reduces both order parameters as may be expected for a simple superposition of the two effects or if an interesting interplay can be observed. The answer to this question is summarized in Fig. 3, where we plotted the impurity contributions of the two relevant order parameters in the system as we cross the phase boundaries along the trajectory in the inset of Fig. 1. The dominant parameter in the phases with one single order is always reduced, while the other parameter remains unchanged close to zero. However, in the supersolid phase only the superfluid density is strongly reduced, while the solid order is in fact enhanced. It is far from obvious why the vacancy should enhance the solid order in this case, contrary to what we observed in the solid phase. The only explanation of the observed behavior is that the vacancy reduces the superfluid density locally so strongly that the solid order is revived, which is evidence for a microscopic competition between the two order parameters. This result clearly shows that the two order parameters are not in an independent superposition. It is noteworthy that the competition and the total change of the order parameters is strongest close to the second order phase transitions to the superfluid phase.

The local expectation values in Fig. 4 also demonstrate the competition of order parameters locally. The local solid order $\left\langle\hat{S}_{i}^{z} \hat{S}_{j}^{z}\right\rangle$ and $\left\langle\hat{S}_{i}^{z} \hat{S}_{j}^{z} \hat{S}_{k}^{z}\right\rangle$ close to the im-

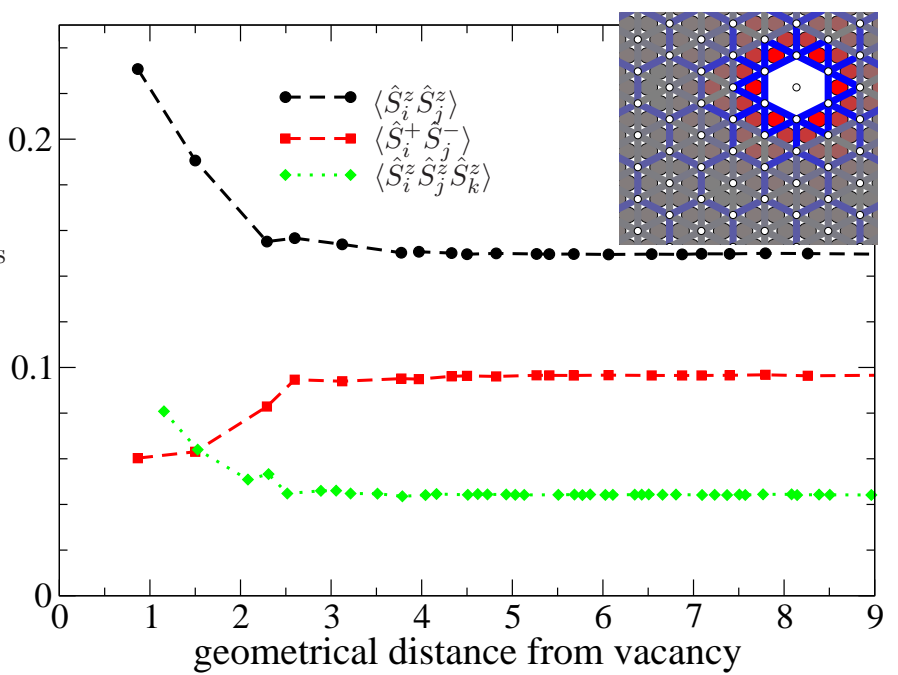

FIG. 4: (color online). Local expectation values as a function of geometrical distance analogous to Fig. 2 in the supersolid phase $(t / V=0.08, B / V=0.2, N=144)$. Closest to the impurity the values almost recover the bulk values in the solid phase in Fig. 2 Inset: Analogous to inset 2 but showing the opposite behavior on a relative color scale.

purity is now enhanced while the kinetic energy $\left\langle\hat{S}_{i}^{+} \hat{S}_{j}^{-}\right\rangle$ is strongly reduced. This is in strong contrast to the observations in the solid phase in Fig. 2 and the relative changes are also much more dramatic and correlated in space, which again demonstrates the direct interplay between both order parameters.

We finally turn to the interesting case of several impurities in the system. A second vacancy on the same sublattice is rather trivial, corresponding to the same pinned order, i.e. constructive interference of the induced magnetization density. Remarkably also a second impurity on an opposite sublattice has the same effect since the second impurity simply lifts the remaining two-fold degeneracy exactly in such a way that both impurities are located on spin-up sublattices. The order is now completely pinned, but all observed effects are approximately additive, i.e. the impurity contributions to the order parameters simply double in the entire parameter space in Fig. 5 Also the observed phase transition between pinned states in Fig. 11remains unchanged. This situation is in sharp contrast to two impurities on different sublat-

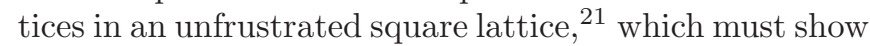
destructive interference of the alternating magnetization.

The most interesting case is given by three impurities on the three different sublattices. The threefold degeneracy of the lattice is then approximately restored again without pinning and the impurities must interfere destructively. This results in a surprisingly strong reduction of the solid order $S(q)$ which now also carries over

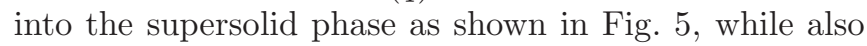
the reduction of $\rho_{s}$ remains strong. Obviously the effects are not simply additive in this case and indicate some interesting impurity-impurity interactions. However, in 


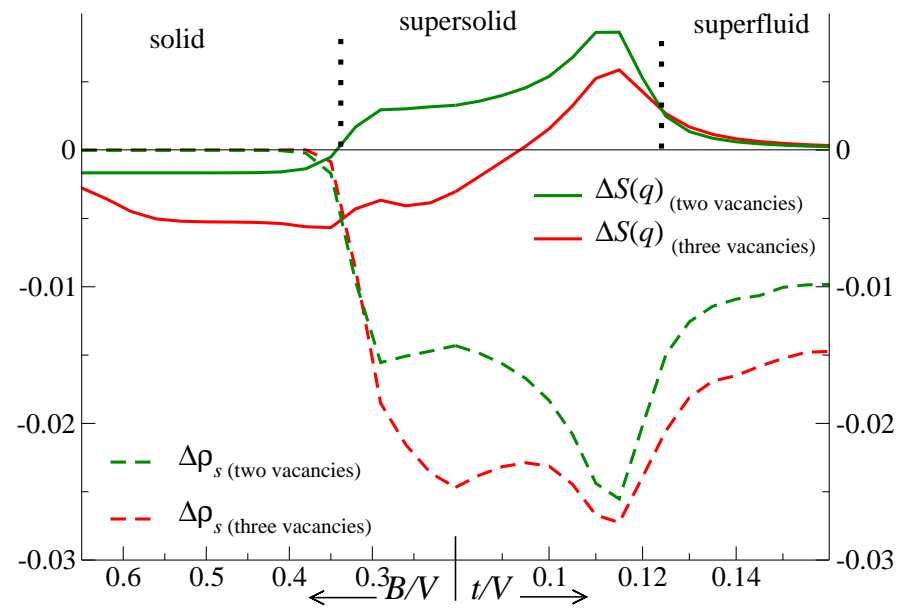

FIG. 5: The impurity contributions to the structure factor and the superfluid density for two and three impurities on different sublattices in a system of 144 sites.

generic systems impurities break the symmetry between the three sublattices, so that the observed pinning and order parameter competition described above is the more general scenario.

In summary, we have used a modified perturbation theory and QMC simulations to analyze impurity effects in a supersolid in comparison to other phases with single order as realized by the model in Eq. (1).

In the solid phase a non-trivial pinning of the entire order by a single defect has been observed. Therefore, impurities create a first order transition line between two different pinned states given by Eq. (4), which is not seen in the pure system.

In the supersolid phase the solid order is surprisingly enhanced by an impurity, which coincides with a strong reduction of the superfluid order. This is evidence for an interesting microscopic competition between the two order parameters, which certainly cannot be in a simple superposition.

For two impurities a simple addition of the observed effects can be seen, while for three impurities on different sublattices a strong destructive interference changes the physics completely. For a more complete understanding of the impurity-impurity interactions on three different sublattices more research is needed.

In all impurity configurations a very strong reduction of the superfluid density $\rho_{s}$ occurs close to the second order supersolid-superfluid transition. It is therefore likely that the superfluid order can be destroyed with a critical density of impurities, while the solid order may be enhanced. The extreme limit of this effect corresponds to the removal of one sublattice, i.e. the honeycomb lattice, which indeed results in an extended solid phase. ${ }^{27}$

The authors would like to thank H.G. Evertz and J.Y. Mao for helpful suggestions and Yue Yu for great support and useful discussions. This work was supported by the National Natural Science Foundation of China under Grants No.10904096 and No.10604024, the Natural Science Foundation of Beijing under Grant No.1092009, the DAAD, and the DFG via the Research Center Transregio 49 .
* Electronic address: zxf@itp.ac.cn

1 G.V. Chester, Phys. Rev. A 2, 256 (1970); A.F. Andreev and I.M. Lifshitz, Sov. Phys. JETP 29, 1107 (1969).

2 E. Kim and M.H.W. Chan, Nature 427, 225 (2004); Science 305, 1941 (2004).

3 A.V. Balatsky and E. Abrahams, J. Supercond. Nov. Magn. 19, 395 (2006).

4 P.W. Anderson, Science 324, 631 (2009).

${ }^{5}$ G. Murthy, D. Arovas, and A. Auerbach, Phys.Rev.B 55, 3104 (1997)

6 S. Wessel and M. Troyer, Phys. Rev. Lett. 95, 127205 (2005); D. Heidarian and K. Damle, Phys. Rev. Lett. 95, 127206 (2005). R.G. Melko, A. Paramekanti, A.A. Burkov, A. Vishwanath, D.N. Sheng, and L. Balents, Phys. Rev. Lett. 95, 127207 (2005).

7 M. Boninsegni and N. Prokof'ev, Phys. Rev. Lett. 95, 237204 (2005).

8 A. Sen, P. Dutt, K. Damle, and R. Moessner, Phys. Rev. Lett. 100, 147204 (2008).

9 F. Wang, F. Pollmann, and A. Vishwanath, Phys. Rev. Lett. 102, 017203 (2009).

10 H.C. Jiang, M.Q. Weng, Z.Y. Weng, D.N. Sheng, and L. Balents, Phys. Rev. B 79, 020409(R) (2009).

11 D. Heidarian and A. Paramekanti, Phys. Rev. Lett. 104, 015301 (2010).
12 L. Pollet, J. D. Picon, H. P. Buchler, and M. Troyer, Phys. Rev. Lett. 104, 125302 (2010).

13 S. Trotzky, P. Cheinet, S. Fölling, M. Feld, U. Schnorrberger, A.M. Rey, A. Polkovnikov, E.A. Demler, M.D. Lukin, and I. Bloch, Science 319, 295 (2008).

14 B. Schmidt, M. Bortz, S. Eggert, M. Fleischhauer and D. Petrosyan, Phys. Rev. A 79, 063634 (2009).

15 C. Becker, P. Soltan-Panahi, J. Kronjäger, S. Dörscher, K. Bongs and K. Sengstock, New J. Phys. 12, 065025 (2010).

16 S. Sachdev, C. Buragohain, and M. Vojta, Science 286, 2479 (1999).

17 A.W. Sandvik and C.J. Hamer, Phys. Rev. B 60, 6588 (1999).

18 K.H. Höglund and A.W. Sandvik, Phys. Rev. Lett. 91, 077204 (2003); Phys. Rev. B 70, 024406 (2004).

19 S. Eggert and I. Affleck, Phys. Rev. Lett. 75, 934 (1995); S. Fujimoto and S. Eggert, Phys. Rev. Lett. 92037206 (2004); J. Sirker, N. Laflorencie, S. Fujimoto, S. Eggert, and I. Affleck, Phys. Rev. Lett. 98137205 (2007).

20 S. Eggert and S. Rommer, Phys. Rev. Lett. 81, 1690 (1998); S. Rommer and S. Eggert, Phys. Rev. B 59, 6301 (1999); Phys. Rev. B 62, 4370 (2000).

21 F. Anfuso and S. Eggert, Phys. Rev. Lett. 96, 017204 (2006). S. Eggert, O.F. Syljuåsen, F. Anfuso, and M. Andres, Phys. Rev. Lett. 99, 097204 (2007). 
22 Vacancies in the hard-core boson model are equivalent only up to an additional symmetry-breaking local field, which is a non-essential complication that is not considered here.

23 E.L. Pollock and D.M. Ceperley, Phys. Rev. B 36, 8343 (1987).

24 A.W. Sandvik, Phys. Rev. B 59, R14157 (1999); O.F. Syljuåsen and A.W. Sandvik, Phys. Rev. E 66, 046701 (2002).
25 P. Sengupta, A.W. Sandvik, and D.K. Campbell, Phys. Rev. B 65, 155113 (2002).

26 R.H. Swendsen and J.-S. Wang, Phys. Rev. Lett. 57, 2607 (1986).

27 S. Wessel, Phys. Rev. B 75, 174301 (2007); J.Y. Gan, Y.C. Wen, J. Ye, T. Li, S.J. Yang, and Y. Yu, Phys. Rev. B 75, 214509 (2007). 